\title{
Sleep problems in Alzheimer's disease: does drug treatment help or harm? ${ }^{\dagger}$
} COMMENTARY ON... COCHRANE CORNER

\author{
Katharine Smith \& Mary-Jane Attenburrow
}

\begin{abstract}
SUMMARY
Drug treatments are commonly used for sleep disturbance in Alzheimer's disease, although none have a specific licence for this indication. This month's Cochrane review assessed the available evidence of benefit or harm in the use of these medications. The review identified two studies of melatonin, which did not show significant improvement in sleep over placebo. One study of trazodone suggested a beneficial effect on sleep, but its small sample size limits the generalisability of the results. Larger studies are needed, with careful assessment of the evidence for possible improvements in sleep but also of important sideeffects such as falls and increased confusion.

DECLARATION OF INTEREST

None
\end{abstract}

\section{Clinical setting}

Forty-four million people worldwide are living with dementia, and with a rapidly ageing population, this number is predicted to nearly double every 20 years (Lancet 2014). The global economic cost is estimated at US $\$ 604$ billion (1\% of global gross domestic product) and the social burden of the disease is undeniable. Alzheimer's disease is the most common type of dementia, accounting for $60-80 \%$ of cases (Alzheimer's Association 2014). It is a neurodegenerative disorder defined by progressive memory loss and cognitive impairment and pathological changes that include the presence of neurofibrillary tangles composed of hyperphosphorylated tau protein and insoluble beta-amyloid plaques associated with activated microglia (Nelson 2012).

At least 25-35\% of people with Alzheimer's disease experience sleep disturbance (Dauvilliers 2007), resulting in significant distress and burden for patients and carers as well as increased healthcare costs and rates of institutionalisation (Gaugler 2000). Common symptoms include night-time sleep fragmentation, increased sleep latency, decreased slow-wave sleep, increased daytime napping and 'sundowning' (Box 1) (Vitiello 1992).
Underlying disease processes in Alzheimer's disease may account for sleep disturbance by affecting maintenance of the circadian rhythm, which is thought to involve the suprachiasmatic nucleus ( $\mathrm{SCN}$ ) in the hypothalamus. In Alzheimer's disease a progressive deterioration in the SCN may cause fluctuations in neurohormones that are critical in the homeostatic maintenance of the circadian rhythm (Wu 2007).

Clinicians are familiar with the burden associated with sleep disturbance in Alzheimer's disease and are commonly asked for treatment advice (once other treatable causes such as intercurrent infection have been excluded), especially when the sleep problem results in high-risk behaviour (such as wandering at night). Non-pharmacological treatments have been used (Deschenes 2009), but studies of these treatments, including bright light, have shown no effect (Forbes 2014).

\section{Choice of treatment}

Currently, there are no medications specifically licensed for sleep disturbance in Alzheimer's disease. Medications for Alzheimer's disease itself (acetylcholinesterase inhibitors such as donepezil) have little effect on sleep disturbance. Therefore, through clinical necessity, but without an informed evidence base, drugs are frequently used empirically on the basis of clinical observation rather than evidence from clinical trials (Deschenes 2009). Sedating antidepressants, particularly trazodone, are prescribed for sleep disturbance in patients with

\section{B0X 1 Sleep terms}

Sleep latency: the time from 'lights out' to sleep onset Total sleep time (TST): actual sleep in a sleep period; equal to total sleep period less movement and awake time; usually expressed as nocturnal TST and daytime TST

Sleep efficiency: the ratio of TST to the amount of time spent in bed

Sundowning: increased confusion, wandering and agitation in the late afternoon and evening
ROUND THE CORNER
Katharine Smith is an honorary consultant psychiatrist at the National Institute for Health Research (NIHR) Oxford cognitive health Clinical Research Facility and Oxford University Department of Psychiatry. She has dual accreditation as a consultant in both general adult and old age psychiatry. Mary-Jane Attenburrow is Clinical Lead at the NIHR Oxford cognitive health Clinical Research Facility, a Senior Research Fellow at Oxford University Department of Psychiatry and an honorary consultant psychiatrist.

Correspondence Dr Katharine Smith, NIHR Oxford cognitive health Clinical Research Facility, Warneford Hospital, Oxford OX3 7JX, UK. Email: katharine.smith@psych.ox.ac.uk

${ }^{\dagger}$ See p. 218 , this issue. 
Alzheimer's disease, but have significant potential for adverse effects (Coupland 2011). Sedativehypnotic agents, including benzodiazepines, have some effect on sleep (decreasing sleep latency and increasing total sleep time (TST); Box 1), but they have little effect on sleep maintenance, which is most commonly affected in older adults. They also carry an increased risk of adverse events in older people, especially falls and cognitive impairment (Glass 2005). The newer-generation non-benzodiazepines, including zolpidem, zaleplon and zopiclone ('Z-drugs'), have shorter half-lives and fewer sideeffects, but data for their use in dementia in older adults are lacking. Antipsychotics with sedative effects, such as quetiapine or olanzapine, may also be used for disturbed sleep in Alzheimer's disease, especially if accompanied by agitated behaviour at night, but their use in dementia has been linked to serious adverse events, including increased mortality. An independent report commissioned and funded by the Department of Health (Banerjee 2009) strongly recommended avoiding the use of atypical antipsychotics in dementia because the risks outweigh the benefits. If antipsychotics are to be used for severe behavioural disturbance, which will probably include insomnia, they should not be given for more than 6 weeks at a time.

Melatonin and melatonin receptor agonists such as ramelteon are used to treat insomnia in healthy people and there has been interest in their use in people with dementia. Melatonin is sold as a dietary supplement in the USA, but in Europe a sustainedrelease form is licensed as a medicine for the shortterm treatment of primary insomnia in adults aged 55 and over. Ramelteon is a melatonin receptor agonist and has a licence in the USA for the longterm treatment of sleep-onset insomnia.

\section{B0X 2 Publication bias}

McCleery et al identified studies that had been started, but either not completed or completed but not published. This raises the possibility of 'publication bias'.

Publication bias describes the preferential reporting that occurs as a result of the tendency for authors, reviewers and editors to publish preferentially those articles that have a clearly defined, statistically significant result. Studies where the treatment has a similar or lesser effect than placebo or than the current well-established treatment are less likely to be published.

In the case of medications for sleep problems in Alzheimer's disease, it is possible that studies were not reported either because the results were not positive for the active drug, or because the harms associated with the drug were equal to or greater than its benefits, or because the researchers failed to recruit the minimum number of patients.

Because there is the potential for significant harm, the possibility of non-reporting of negative studies is particularly important. Note that the reviewers in this case did make strenuous efforts to obtain the unpublished data but were not able to. It will be important for updaters of this review to make similar rigorous efforts to obtain unpublished data and to report whether or not they were successful in this.

\section{Results of the review}

McCleery et al searched (updated in March 2013) for randomised controlled trials (RCTs) involving people with Alzheimer's disease who had a sleep problem diagnosed on subjective or objective measures. They found five completed RCTs. There were also two ongoing trials (of melatonin and zolpidem) with no results yet available. In addition, a number of relevant trials were identified but, despite rigorous efforts from the reviewers, no data could be obtained (see Box 2 for implications).

\section{Melatonin}

Three studies were included in the review, but the reviewers could obtain usable data for analysis from only two. (The third trial reported no benefit on sleep or adverse effects with melatonin, but presented only summary data for some outcomes and did not specify the method used for analysis.) Data from the two trials revealed no significant effect of melatonin on nocturnal TST $(n=184$, mean difference $($ m.d. $)=10.68$ minutes, $95 \%$ CI -16.22 to 37.59$)$ or on the ratio of daytime sleep to night-time sleep $(n=184$, m.d. $=-0.13,95 \%$ CI -0.29 to 0.03 ). Adverse effects (including symptoms such as falls and fatigue) did not differ significantly between melatonin and placebo groups in number ( $n=151$, m.d. $=0.20,95 \%$ CI -0.72 to 1.12 ; one study) or severity (3-point scale from $1=$ mild to $3=$ severe; m.d. $=0.10,95 \% \mathrm{CI}-0.06$ to 0.26 ; one study). Carer-rated sleep quality, cognition (measured on the Mini Mental State Examination and the Alzheimer's Disease Assessment Scale - Cognition) and activities of daily living did not differ significantly between groups. Quality of life and carer burden were not assessed.

\section{Trazodone}

Data came from one small study $(n=30)$ (Camargos 2014). The trazodone group had longer nocturnal TST and greater sleep efficiency (Box 1). The two groups did not differ significantly on other measures of sleep quality. Adverse events, although not described, were similar in number and severity in both groups.

\section{Ramelteon}

Data come from one trial $(n=74$, only 66 completed), reported in summary on the sponsor's website. There was no evidence of benefit from ramelteon over placebo in the treatment of sleep disturbances in patients with mild to moderate Alzheimer's disease. There was weak evidence, incompletely reported, of more daytime sedation in the ramelteon group. 


\section{Implications for clinical practice}

Even if there is a lack of clear evidence to help guide drug treatment of sleep problems in Alzheimer's disease, some evidence to inform practice can be drawn from McCleery et al's review. Adequately reported data were available for only two drugs. Trazodone is commonly prescribed for this indication, and the study that shows that a low dose $(50 \mathrm{mg})$ of trazodone improves sleep problems in Alzheimer's disease is a welcome addition to the evidence base. However, it is a very small study and therefore the results are preliminary. Trazodone has a well-described sideeffect profile when used (albeit at higher doses) as an antidepressant (Coupland 2011). In this small study, however, there may have been insufficient power to detect side-effects such as falls and confusion. The studies of melatonin identified in this review suggest that melatonin is unlikely to be of benefit to patients with Alzheimer's disease and sleep problems.

Although there are two small ongoing studies (NCT00940589 on melatonin and NCT00814502 on zolpidem), and another small study has reported in abstract form since the review (NCT01867775 on mirtazapine: $n=24$, no significant benefit on sleep), larger pragmatic trials are needed in this area. At present, organisations such as the National Institute for Health and Care Excellence (NICE 2015) are unable to give guidance on pharmacological treatment because of the lack of reliable, large-scale evidence of potential benefit.

Assessment of harm cannot be ignored. Behavioural disturbance in dementia is often treated with drugs. We already know that the prescription of antipsychotics in dementia carries an associated increase in adverse events and mortality (Ballard 2014) and should be minimised where possible. The National Dementia and Antipsychotic Prescribing Audit (Health and Social Care Information Centre 2012) showed a fall of $51.8 \%$ in the number of people with dementia receiving a prescription of antipsychotic medication between 2008 and 2011. There was a similar but smaller reduction in antidepressant prescriptions in dementia. However, almost unnoticed in this is the use of hypnotics. Figures were not reported separately in the audit, but international rates of hypnotic usage are high, particularly in care homes (typically ranging from 22 to 47\%; Connelly 2013). We need to be careful that as clinicians we have not moved from using one potentially toxic drug to another, without an adequate evidence base for relative benefit versus harm.

\section{Acknowledgements}

The authors acknowledge support from the NIHR Oxford cognitive health Clinical Research Facility and thank Professors Robin Jacoby and John Geddes, and Associate Professor Andrea Cipriani, Oxford University Department of Psychiatry, for their comments and suggestions. The views expressed are those of the authors and not necessarily those of the NHS, the NIHR or the Department of Health.

\section{References}

Alzheimer's Association (2014) Alzheimer's Association Report 2014: Alzheimer's disease facts and figures. Alzheimer's \& Dementia, 10: e47-92.

Ballard C, Corbett A, Howard R (2014) Prescription of antipsychotics in people with dementia. British Journal of Psychiatry, 205: 4-5.

Banerjee S (2009) The Use of Antipsychotic Medication for People with Dementia: Time for Action. A Report for the Minister of State for Care Services. Department of Health.

Camargos EF, Louzada LL, Quintas JL, et al (2014) Trazodone improves sleep parameters in Alzheimer disease patients: a randomized, doubleblind, and placebo-controlled study. American Journal of Geriatric Psychiatry, 22: 565-74

Connelly P (2013) Hypnotic use in people with dementia - are our eyes open to the problem? Old Age Psychiatrist, 57: 1-5.

Coupland C, Dhiman P, Morriss R, et al (2011) Antidepressant use and risk of adverse outcomes in older people: population based cohort study. BMJ, 343: d4551.

Dauvilliers $Y$ (2007) Insomnia in patients with neurodegenerative conditions. Sleep Medicine, 4 (suppl 4): S27-34.

Deschenes CL, McCurry SM (2009) Current treatments for sleep disturbances in individuals with dementia. Current Psychiatry Reports, 11: 20-6.

Forbes D, Blake CM, Thiessen EJ, et al (2014) Light therapy for improving cognition, activities of daily living, sleep, challenging behaviour, and psychiatric disturbances in dementia. Cochrane Database of Systematic Reviews, 2: CD003946.

Gaugler JE, Edwards AB, Femia EE, et al (2000) Predictors of institutionalization of cognitively impaired elders: family help and the timing of placement. Journals of Gerontology. Series B, Psychological Sciences and Social Sciences, 55: 247-55.

Glass J, Lanctot KL, Herrmann N, et al (2005) Sedative hypnotics in older people with insomnia: meta-analysis of risks and benefits. BMJ, 331: 1169.

Health and Social Care Information Centre (2012) National Dementia and Antipsychotic Prescribing Audit. HSCIC.

Lancet (2014) Addressing global dementia. Lancet, 383: 2185.

McCleery J, Cohen DA, Sharpley AL (2014) Pharmacotherapies for sleep disturbances in Alzheimer's disease. Cochrane Database of Systematic Reviews, 3: CD009178.

National Institute for Health and Care Excellence (2015) Dementia: Supporting People with Dementia and their Carers in Health and Social Care (issued: November 2006; last modified: March 2015) (NICE Clinical Guideline 42). NICE.

Nelson PT, Alafuzoff I, Bigio EH, et al (2012) Correlation of Alzheimer disease neuropathologic changes with cognitive status: a review of the literature. Journal of Neuropathology and Experimental Neurology, 71: 362-81.

Vitiello M, Bliwise D, Prinz P (1992) Sleep in Alzheimer's disease and the sundown syndrome. Neurology, 42 (suppl 6): 83-94.

Wu YH, Swaab DF (2007) Disturbance and strategies for reactivation of the circadian rhythm system in aging and Alzheimer's disease. Sleep Medicine, 8: 623-36. 